\section{AB0115 SEMAPHORIN 4A PERPETUATE SYNOVIAL FIBROBLAST INTERACTIONS WITH CD4+ T CELLS AND OSTEOCLAST DIFFERENTIATION: IMPLICATION IN RHEUMATOID ARTHRITIS}

Zhu Chen, Yuanyuan Zhang, Xu Zheng, LI Xiaomei. University of Science and Technology of China, Hefei, China

Background: Rheumatoid arthritis synovial fibroblast (RASF) and T cells are important contributors in the pathogenesis of RA. Semaphorin 4A has been reported to be elevated in $R A$ patients and play crucial role in promoting inflammation of RA. However, whether semaphorin 4A could facilitate RASF interactions with $\mathrm{CD}^{+} \mathrm{T}$ cells and osteoclastogenesis is less known.

Objectives: The present study aims to investigate the role of semaphorin $4 \mathrm{~A}$ Plexin $\mathrm{B} 1$ axis in RASF interactions with $\mathrm{CD}^{+} \mathrm{T}$ cells and osteoclastogenesis in vitro.

Methods: Mouse synovial fibroblasts (SF) were isolated from collagen-induced arthritic mice and cultured in vitro with TNF- $\alpha$ and IL- $\beta$ for $24 \mathrm{~h}$. Small interfering RNA (siRNA) against Semaphorin 4A and Plexin B1 were constructed and transfected into SF via adenovirus. Splenic $\mathrm{CD}^{+} \mathrm{T}$ cells isolated from arthritic mice were cocultured with SF under anti-CD3 and anti-CD28. The proportion of $\mathrm{CD}^{+}{ }^{+} \mathrm{LL}-17^{+}$Th17 cells was determined at day 5 . PBMC isolated from blood of healthy donors were incubated with $1 \mu \mathrm{g} / \mathrm{ml}$ Plexin D1 neutralizing antibody overnight. Cells were washed for monocyte enrichment and cultivated for 14 days in $\alpha-$ MEM supplemented with MCSF and RANKL. Osteoclast differentiation was evaluated by TRAP staining. Total RNA was extracted and expression of osteoclast markers were examined by quantitative real-time PCR

Results: Adenovirus-Mediated siRNA efficiently inhibited expression of Semaphorin 4A and Plexin B1 in SF. The proportion of Th17 cells was significantly decreased in both Semaphorin 4A and Plexin B1 transfected SF cocultures. The number of Trap positive osteoclasts were significantly decreased in cultures that pretreated with Plexin D1. Consistently, blocking of Plexin D1 signaling dramatically downregulated mRNA expression level of Trap, Cathepsin K and NFATc1. Conclusion: Semaphorin $4 \mathrm{~A}$ perpetuate synovial fibroblasts interactions with $\mathrm{CD}^{+} \mathrm{T}$ cells by promoting Th17 differentiation. Moreover, Semaphorin 4A-Plexin D1 axis promote osteoclastogenesis and therefore might serve as a potential therapeutic target in the treatment of RA.

\section{REFERENCES}

[1] lyer AS, et al. Neuroimmune Semaphorin 4A in Cancer Angiogenesis and Inflammation: A Promoter or a Suppressor? Int J Mol Sci. 2018 Dec 30;20 (1).

[2] Chapoval SP, et al. Semaphorins $4 A$ and $4 D$ in chronic inflammatory dis eases. Inflamm Res. 2017 Feb;66(2):111-117

[3] Wang L, et al. Expression of Semaphorin 4A and its potential role in rheumatoid arthritis. Arthritis Research \& Therapy (2015) 17:227.

Disclosure of Interests: None declared

DOI: 10.1136/annrheumdis-2019-eular.2318

\section{AB0117 HOMOCYSTEINYLATED ALPHA 1 ANTI-TRYPSIN AS A POTENTIAL ANTIGENIC TARGET IN RHEUMATOID ARTHRITIS}

Tania Colasanti ${ }^{1}$, Danilo Sabatinelli ${ }^{1}$, Carmine Mancone ${ }^{2}$, Arbi Pecani $^{3}$ Mariangela Speziali ${ }^{1}$, Marta Vomero ${ }^{1}$, Cristiana Barbati ${ }^{1}$, Alessandra Ida Celia ${ }^{1}$, Annacarla Finucci ${ }^{1}$, Carlo Perricone ${ }^{1}$, Fulvia Ceccarelli ${ }^{1}$, Francesca Spinelli ${ }^{1}$, Vincenzo Barnaba ${ }^{1}$, Fabrizio Conti ${ }^{1}$, Guido Valesini ${ }^{1}$, Cristiano Alessandri ${ }^{1}$. ${ }^{1}$ Sapienza University of Rome, Department of Internal Medicine and Medical Specialties, Roma, Italy; ${ }^{2}$ Sapienza University of Rome, Department of Molecular Medicine, Roma, Italy, ${ }^{3}$ University Hospital "Shefqet Ndroqi", Department of Internal Medicine, Tiranë, Albania

Background: Rheumatoid arthritis (RA) is a chronic, inflammatory, autoimmune disorder that primarily affects joints. Beside the well-known rheumatoid factor (RF) and anti-citrullinated protein antibodies (ACPA) have been reported to be a very useful diagnostic and prognostic marker of RA, recently antibodies against carbamylated proteins (anti-CarP) have been described also in ACPA negative RA patients. However, more than $20 \%$ of RA cases are still defined as seronegative forms. Therefore, the individuation of new antibody specificities in RA could be helpful for diagnostic and prognostic purposes.

Objectives: The goals of this study were the identification and the immunologic characterization of post-translational modified synovial fluid (SF) autoantigens, specifically targeted by autoantibodies from sera of seronegative RA patients. Methods: SFs from 5 seronegative RA patients were collected, pooled and treated with hyaluronidase. After removal of both albumin and $\operatorname{lgG}$, the sample was washed and cleansed, concentrated, separated by 2-dimensional electrophoresis (2-DE) and then transferred by Western blotting to nitrocellulose membrane, for autoantigen detection by immunoassay, using a pool of sera from 5 seronegative RA patients. The antigenic protein spots were identified by peptide mass fingerprint, using a Matrix-Assisted Laser Desorption/lonization-Time O Flight (MALDI-TOF) mass spectrometer.

This approach revealed Alpha 1 Anti-Trypsin (A1AT) as a target of RA patients autoantibodies.

Pooled SFs were also analyzed by reverse-phase nanoliquid chromatography and tandem mass spectrometry, to confirm the presence of A1AT and for the identification of A1AT post-translational modifications.

Homocysteinylated A1AT was immunoprecipitated from pooled SFs of RA seronegative patients and, after a diafiltration and concentration process, was used as an antigen to detect anti-homocysteinylated A1AT antibodies by Enzyme-Linked ImmunoSorbent Assay (ELISA). In order to this, consecutive patients with RA osteoarthritis (OA), psoriatic arthritis (PsA), and healthy donors were enrolled and sera were collected.

Results: Homocysteinylated A1AT was identified as a potential antigenic target not only in ACPA and RF positive RA patients but, more importantly, also in RA seronegative patients.

Antibodies anti-homocysteinylated A1AT were found in $66.7 \%(44 / 66)$ RA patients seronegative for ACPA and RF, 88.6\% (39/44) RA patients, 15\% (3/20) OA patients, $26.3 \%(5 / 19)$ PsA patients and in none (0/41) of healthy donors.

Conclusion: Homocysteinylated A1AT was identified as a new possible antigenic target of autoantibodies in sera from RA patients, including RA seronegative patients. This tool may be useful in diagnosis and monitoring of the disease and may contribute to understand the immunopathogenic mechanisms of RA in future studies.

Disclosure of Interests: Tania Colasanti: None declared, Danilo Sabatinelli: None declared, Carmine Mancone: None declared, Arbi Pecani: None declared, Mariangela Speziali: None declared, Marta Vomero: None declared, cristiana bar bati: None declared, Alessandra Ida Celia: None declared, Annacarla Finucci: None declared, Carlo Perricone Speakers bureau: BMS; Lilly, Celgene, Sanofi, Fulvia Ceccarelli: None declared, francesca spinelli: None declared, Vincenzo Barnaba: None declared, fabrizio conti: None declared, Guido Valesini: None declared, cristiano alessandri: None declared

DOI: 10.1136/annrheumdis-2019-eular.3012

\section{AB0119 1 MECHANISM OF NEUTROPHIL EXTRACELLULAR TRAP IN PROMOTING SYNOVIAL HYPERPLASIA IN RHEUMATOID ARTHRITIS}

Zhang Hui ${ }^{1}$, Feng Yuchen ${ }^{2}$, Kang Guorong ${ }^{2}$, Liu Jinwu ${ }^{2}$, Shen HaiLi ${ }^{3}$. ${ }^{1}$ Second Clinical Medical College, Lanzhou University, Lanzhou, China; ${ }^{2}$ Second Clinical Medical College, Lanzhou University, Lanzhou, China: ${ }^{3}$ Department of Rheumatology, Lanzhou University Second Hospital, LanZhou, China

Background: Rheumatoid arthritis(RA)is an autoimmune disease characterized by chronic inflammation of the synovial membrane and pannus formation. With the discovery of Neutrophil extracellular traps (NETs) in 2004, its role in autoimmune diseases has received much attention ${ }^{[1]}$.At present,studies on the pathogenesis of NETs in RA have focused on NETs as a source of citrullinated antigens ${ }^{[2]}$, producing autoantibodies to citrullinated protein antigens (ACPAs) ${ }^{[3]}$. In addition, NETs can activate rheumatoid arthritis fibroblast-like synoviocytes (RA-FLSs) to produce inflammatory cytokines ${ }^{[2]}$.Studies have reported that connective tissue growth factor (CTGF) is overexpressed in RA serum ${ }^{[4]}$, and CTGF plays a key role in the proliferation of RA-FLSs, furthermore leads to joint destruction in RA patients ${ }^{[5]}$. Therefore, our study stimulated RA-FLSs with NETs to detect cell proliferation and CTGF mRNA, aiming to explore the pathogenesis of NETs involved in RA.

Objectives: To Explore the potential effects of Neutrophil extracellular traps (NETs) on rheumatoid arthritis synovial fibroblasts (RA-FLSs).

Methods: The synovial tissues of RA patients were isolated and cultured in vitro peripheral blood neutrophils were extracted from healthy volunteers and stimulated to formation NETs, NETs were extracted as a stimulating agent; MTS prolif eration assay was used to evaluate the effect of NETs on the proliferation of RA FLSs; qRT-PCR was used to determine the expression of connective tissue growth factor (CTGF) mRNA in cells treated with NETs-stimulated RA-FLSs for $60 \mathrm{~h}$.

Results: The isolated and purified neutrophils could form NETs by stimulated in vitro. The concentration of extracted NETs -DNA was $58.5 \mathrm{ng} / \mathrm{ul}\left(1 \times 10^{6}\right.$ cells) Compared with the control group, NETs could promote the proliferation of RAFLSs. With the increase of NETs concentration, the proliferation of RA-FLSs was also enhanced( $\mathrm{F}=99.519, \mathrm{P}<0.05)$;NETs stimulated the up-regulation of CTGF mRNA expression in RA-FLSs $(30.696 \pm 0.468$ vs. $1, p<0.01)$

Conclusion: NETs can promote the proliferation of RA-FLSs and stimulate the up-regulation of CTGF mRNA in RA-FLSs in vitro. 


\section{REFERENCES}

[1] April Barnado, Leslie J. Crofford, Jim C. Oates. At the Bedside: Neutrophil extracellular traps(NETs) as targets for biomarkers and therapies in autoimmune diseases. Journal of Leukocyte Biology Vol.99, No.2, pp:265-278, July, 2017.

[2] Khandpur R, Carmona-Rivera C, Vivekanandan-Giri A, et al. NETs are a source of citrullinated autoantigens and stimulate inflammatory responses in rheumatoid arthritis. Sci Transl Med (2013) 5(178):178ra40.

[3] Corsiero E, Bombardieri M, Carlotti E,Pitzalis C, et al. Single cell cloning and recombinant monoclonal antibodies generation from RA synovial B cells reveal frequent targeting of citrullinated histones of NETs.Ann Rheum Dis 2016;75:1866-1875.

[4] Nozawa K, Fujishiro M, Kawasaki M, et al. Connective tissue growth factor promotes articular damage by increased osteoclastogenesis in patients with rheumatoid arthritis. Arthritis Res Ther. 2009;11(6):R174.

[5] S Ding, H Duan, F Fang, et al.CTGF promotes articular damage by increased proliferation of fibroblast-like synoviocytes in rheumatoid arthritis. Scand J Rheumatol 2016;45:4,282-287.

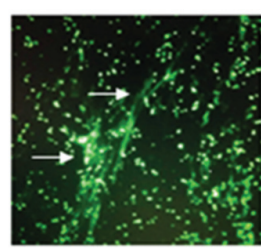

$(\times 40)$

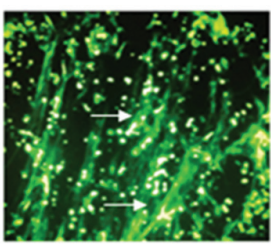

$(\times 100)$
Fig.1.Identification of NETs by fluorescent staining

PicoGreen fluorescent staining showed extracellular fibrous network structure
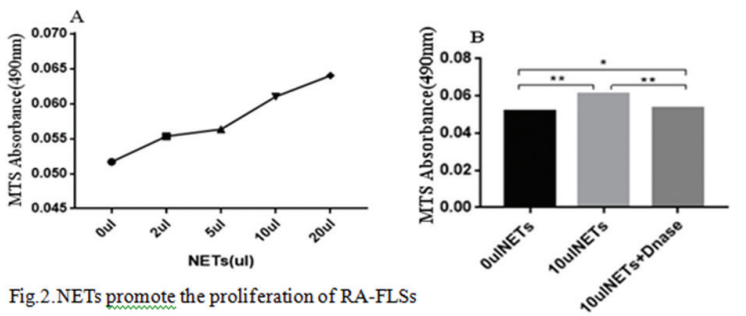

Fig.2.NETs promote the proliferation of RA-FLS

(A)Different doses of NETs stimulated RA-FLSs, and the OD value at $490 \mathrm{~nm}$ was detected after $60 \mathrm{~h}$ culture.(B)The OD values of NETs group (10ul NETs) and DNaseI pretreated NETs group (DNaseI+10ul NETs) were higher than the control group (Oul NETs). ( $\left.{ }^{*} \mathrm{P}<0.05, * * \mathrm{P}<0.01\right)$

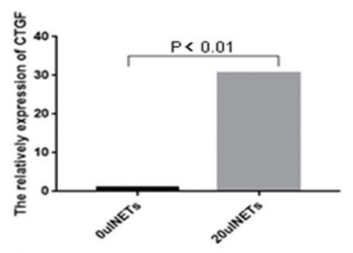

Fig.3.CTGF mRNAlevels in RA-FLSs

The relative expression of CTGF mRNA in RA-FLSs between experimental group (20ul NETs) and control group (Oul NETs).

Disclosure of Interests: None declared

DOI: 10.1136/annrheumdis-2019-eular.772

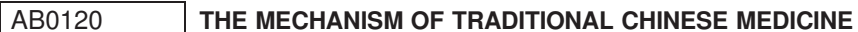 PRESCRIPTION ER-MIAO-SAN IN THE TREATMENT OF RHEUMATOID ARTHRITIS BASED ON CHOLINERGIC ANTI-INFLAMMATORY PATHWAY}

Ze Wang, Huiqin Hao, LI Zhen, Yuting Gao, Jianhong Guan. SHANXI UNIVERSITY OF CHINESE MEDICINE, Basic Laboratory of Integrated Traditional Chinese and Western Medicine, Shan Xi, China

Background: Rheumatoid arthritis (RA) is a chronic progressive autoimmune disease. The traditional Chinese herbal formula Er-miao-san (EMS) has been used to treat RA demonstrating significant clinical efficacy; however, the mechanism of action remains unclear. In view of the important role of $\alpha 7$ nicotinic acetylcholine receptor $(\alpha 7 \mathrm{nAChR})$ in the cholinergic anti-inflammatory pathway (CAP)for the regulation of inflammation and cytokines. Indeed, we previously found a correlation between CHRNA7 (encoding $\alpha 7 n A C h R$ ) expression and EMS, we hypothesized that it may play a role in the anti-inflammatory effects of EMS

Objectives: The mechanism of EMS in the treatment of RA based on CHRNA7 involved in the regulation of CAP.

Methods: We established a CIA model with female Wistar, and the effects of intragastric administration of EMS on the expression of CHRNA7, arthritis score, inflammatory, and articular cartilage changes, was examined in the joints. The serum levels of TNF- $\alpha$, IL- 6 , and IL- $1 \beta$ were determined using commercial ELISA kit.

Results: The CIA model was successfully established. Macroscopic changes of arthritis, such as redness and swelling, were clearly observed in the CIA rats, but were attenuated by the treatment of EMS. The mean arthritis score was markedly lower in the EMS-treated group (EG, $P<0.05$ ). The serum level of TNF- $\alpha$ was significantly lower in EG compared with $\mathrm{CIA}$ group $(P<0.05)$. The same results were found in the serum levels of IL- 6 and IL-1 13 . Synovial edema and extensive infiltration of inflammatory cells occurred in the CIA rats, but were repaired by the treat ment of EMS. Cartilage tissue was thinning, dissolution and disappearance, as well as extensive inflammatory cell infiltration with plasma cells and lymphocytes, was observed in the articular cartilage of the ankles in CIA group. In contrast, EMS treatment prevented cartilage degeneration and markedly reduced inflammation. Immunohistochemistry $(\mathrm{IHC})$ analysis showed positive signals of CHRNA7 was expressed on fibroblast-like synoviocytes, macrophages, and endothelial cells in the joints. Effect of EMS on the expression of CHRNA7 protein in the joint by Western blot (WB) analysis. IHC and WB relative optical density values of CHRNA7 was significantly higher in EG compared with CIA group ( $P$ $0.05)$.

Conclusion: EMS can significantly alleviate the symptoms of arthritis in CIA rats by regulating the expression of CHRNA7 in CAP. It provides a scientific research foundation for the further development of EMS and explores more ways to treat RA.

\section{REFERENCES}

[1] Lam F. F., Ko I. W., Ng E. S., et al. (2008). Analgesic and anti-arthritic effects of Lingzhi and San Miao San supplementation in a rat model of arthritis induced by Freund's complete adjuvant. J Ethnopharmacol, 120 (1), 44-50.

[2] Chen G., Li K. K., Fung C. H., et al. (2014). Er-Miao-San, a traditional herbal formula containing RhizomaAtractylodis and Cortex Phellodendri inhibits inflammatory mediators in LPS-stimulated RAW264. 7 macrophages through inhibition of NF-KB pathway and MAPKs activation. J Ethnopharmacol, 154(3), 711-718.

[3] Wang H., Yu M., Ochani M., et al. (2003). Nicotinic acetylcholine receptor alpha7 subunit is an essential regulator of inflammation. Nature, $421, \mathrm{~N}$ 6921, 384-388

[4] Kanashiro A., Talbot J., Peres R. S., et al. (2016). Neutrophil Recruitment and Articular Hyperalgesia in Antigen-Induced Arthritis are Modulated by the Cholinergic Anti-Inflammatory Pathway. Basic \& clinical pharmacology \& toxicology, 119(5), 453-457.

[5] Li S., Zhou B., Liu B., et al. (2016). Activation of the cholinergic anti-inflammatory system by nicotine attenuates arthritis via suppression of macrophage migration. Molecular medicine reports, 14(6), 5057-5064.

Disclosure of Interests: None declared

DOI: 10.1136/annrheumdis-2019-eular.4774

\section{AB0121 EXPRESSION OF CHEMOKINES AND CHEMOKINE RECEPTORS IN DIFFERENT TISSUES AND THEIR LOCALIZATION IN THE JOINTS OF RATS WITH RHEUMATOID ARTHRITIS}

LI Zhen ${ }^{1}$, Huiqin $\mathrm{Hao}^{1,2}$, Ze Wang ${ }^{2}$, Wenjing Lu ${ }^{2}$, Yang Liu ${ }^{1}$, on behalf of Innovation Team of Chinese and Western Medicine Combined with Inflammatory Joint Disease Research at Shanxi University of Chinese Medicine. ${ }^{1}$ Shanxi University of Chinese Medicine, College of Basic Medical Sciences, Jinzhong, China; ${ }^{2}$ Shanxi University of Chinese Medicine, Basic Laboratory of Integrated Traditional Chinese and Western Medicine, Jinzhong, China

Background: Rheumatoid arthritis (RA) is a complex, chronic, multisystem autoimmune disease characterized by a sustained immune response that leads to inflammation in the body and destruction of joints. While not completely understood, immune cells, as well as soluble factors such as cytokines and chemokines, are believed to be involved in the pathogenesis of RA. chemokines play an important role in the development of inflammation and the regulation of cytokines. However, studies of chemokines have focused mainly on their role in cancer, and 